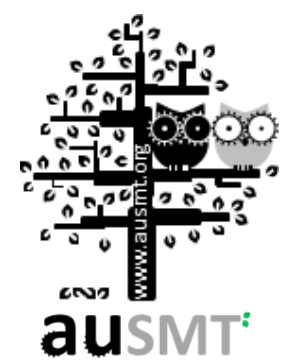

\title{
Controlling a Rehabilitation Robot with Brain-Machine Interface: An approach based on Independent Component Analysis and Multiple Kernel Learning
}

\author{
Yi-Hung Liu ${ }^{1}$, , Han-Pang Huang ${ }^{2}$, Tzu-Hao Huang2 \\ Zhi-Hao Kang ${ }^{2}$ and Jyh-Tong Teng 1 \\ ${ }^{1}$ Department of Mechanical Engineering, Chung Yuan Christian University, Taiwan \\ ${ }^{2}$ Department of Mechanical Engineering, National Taiwan University, Taiwan \\ (Received 15 November 2012; Accepted 1 December 2012; Published on line 1 March 2013) \\ *Corresponding author: jlyh@cycu.edu.tw \\ DOI: 10.5875 /ausmt.v3i1.175
}

\begin{abstract}
Patients suffering from severe motor disabilities are usually dependent on assistance from other people to engage in rehabilitation exercises, making the rehabilitation process time-consuming and inconvenient. We propose an automatic feature extraction method for a brain-machine interface that allows patients to control a robot using their own brain waves. A brain-machine interface (BMI) based on the P300 event-related potential (ERP), called the Brain Controlled Rehabilitation System (BCRS), was developed to detect patient intentions. Using the BCRS, patients can communicate with the robot through their brain waves. However, obtaining an automatically extracted, useful EEG signal is a difficult and important problem for BMI research. In this paper, Independent Component Analysis Multiple Kernel Learning (ICA-MKL) is used to directly extract a useful signal and build the classification mode for BCRS. The results reveal that this method is useful for automatically extracting the P300 signal and improves on the accuracy of MKL. In addition, the same method can be extended to any motor imagery area. The ICA-MKL approach for brain imagery data also effectively removes eye-blink artifacts.
\end{abstract}

Keywords: brain-machine interface; event-related potential; brain wave; ICA; MKL

\section{Introduction}

Brain-computer interfaces $(\mathrm{BCl})$ have received increasing attention over the past decade. $\mathrm{A} \mathrm{BCl}$ allows a user to communicate with the external environment through the recording and recognition of the user's brain activities/waves [1-3]. Of the various techniques that can be used to measure brain activity, the most popular is the EEG due to advantages including high-temporal resolution, portability, and non-invasive recording. Brain waves commonly used for $\mathrm{BCl}$ include slow cortical potential (SCP), mu rhythm, beta wave, and P300 event-related potential (ERP).

P300 ERP appears as a positive peak in the EEG measured from the central site after the presentation of an infrequent and anticipated stimulus, and typically has a latency of around 300 ms. Based on the P300 ERP, a brain-controlled rehabilitation system (BCRS) is developed to allow users to autonomously control a robot which assists them in engaging in rehabilitation exercise. The BCRS is particular useful for patients suffering severe motor disabilities due to cerebral vascular accidents (CVA), spinal cord injuries (SCI), traumatic brain injuries (TBI), multiple sclerosis (MS), amyotrophic lateral sclerosis (ALS) or Parkinson's disease (PD)[1].

For $\mathrm{BCls}$, one of the critical issues is the acquisition of a clean (or actual) EEG (or actual EEG) acquisition. Due to the noninvasive measuring technique used, the 
Yi-Hung Liu received his M.S. in engineering science and ocean engineering and his Ph.D. degree in mechanical engineering, both from National Taiwan University, Taiwan, in 1996 and 2003, respectively. In 2003, he joined the faculty of Chung Yuan Christian University, Taiwan, where he is currently Associate Professor of Mechanical Engineering. His research interests include machine learning, machine vision, brain-computer/robot interfaces, neuroprostheses, and intelligent system diagnosis.

Dr. Liu is a member of the IEEE and CIAE. He currently serves as Associate Editor of the International Journal of Automation and Smart Technology (AUSMT), and Guest Editor of the Journal of Neuroscience and Neuroengineering (JNSNE) issued by American Science Publishers. He is the current Co-chair of the Technical Committee on Medical Mechatronics of IEEE's SMC society. He has published 60 papers in journal and conference proceedings. He was the recipient of the 2006 Best Paper Award from the Chinese Institute of Industrial Engineers, the recipient of the Annual Best Paper Award from the 2009 Automatic Optical Inspection Forum and Competition, and the recipient of the Best Paper Award from the 2nd International Conference on Mechatronics and Applied Mechanics in 2012. He was named in Marquis' Who's Who in Asia 2007, and Who's Who in the World 2008, 2009, and 2010.

Han-Pang Huang received his Ph.D. in electrical engineering from The University of Michigan, Ann Arbor, in 1986. He is a Distinguished Professor and Zhong Zhuo-Zhang Chair Professor in the Department of Mechanical Engineering and the Graduate Institute of Industrial Engineering, National Taiwan University. He is the Fellow of the CSME and the IEEE PAB, He serves as Editor of IEEE/ASME Trans. on Mechatronics, the Intl. J. of Advanced Robotics Systems, and the International Journal of Electronic Business Management. He is co-author of the books "Fuzzy Neural Intelligent Systems: Mathematical Foundation and the Applications in Engineering," published by CRC Press in January, 2001, "Introduction to Nano Engineering," published by Princeton International Publishing Co., 2003, and "Introduction to Flat Panel Display," published by Princeton International Publishing Co., 2008. Dr. Huang's research interests include intelligent robotic systems, prosthetic hands, manufacturing automation, and control systems. He has published more than 330 papers in refereed technical journals and conference proceedings, and holds more than 21 patents. Dr. Huang is recipient of the Ford University Research Award 1996-1998; National Science Counci Outstanding Research Awards, 1996-1998, 1998-2000, 2000-2002; National Science Council Distinguished Research Awards, 2002-2008; National Science Council Distinguished Research Fellow Awards, 2009, TECO Outstanding Science and Technology Research Achievement Award, 2012, and Distinguished Education Award on RFID from EPCglobal (Taiwan), 2010. He served as Guest Editor of IEEE/ASME Trans. on Mechatronics in 2001 and 2006, and of IEEE Trans. on Industrial Electronics in 2008 and 2009. He served as Editor-in-Chief of the Journal of Chinese Fuzzy System Association from September 1997 to September 1999, and of the International Journal of Fuzzy System from September 1999 to December 2002. He was Associate Editor of IEEE Trans. on Automation Science and Engineering from June 2003 to August 2005, and Board Member of the International Journal of Advanced Robotics from 2004 to 2008, and the MIT Supply Chain Forum 2005-2008. He also served as the President of the Chinese Institute of Automation Engineers, Program Director of the Automation Technology Division, National Science Council; and as Adviser to the Industry Bureau, Department of Technology, and Ministry of Education.

Tzu-Hao Huang received his M.D. in rehabilitation science and technology from Nation Yang Ming University, Taipei, Taiwan in 2006. He is currently a Ph.D. student in the Department of Mechanical Engineering at National Taiwan University. His current research interests include robot hand design and control, biomedical engineering, machine learning, and pattern recognition.

Jyh-Tong Teng has been professor of Mechanical Engineering at Chung Yuan Christian University in Chung-Li, Taiwan since 1989. Prior to joining CYCU, he was a Senior Engineer and Tech Leader at the General Electric Nuclear Energy Business Group. He received his Ph.D. from the Department of Mechanical Engineering at the University of California, Berkeley in 1978.

At CYCU, Professor Teng has served as Departmental Chair of ME, Dean of the Office of International Affairs, and Dean of the College of Engineering, during which the College of Engineering was accredited by the Institute of Engineering Education Taiwan (IEET). He currently serves on the board of the IEET. Professor Teng's areas of research include compartment fire and smoke behavior, thermal management of electronic devices and systems, microchannel thermo-fluids, nuclear power plant safety evaluation and severe accident management.
Zhi-Hao Kang received is a Ph.D. candidate in the Department of Mechanical Engineering at National Taiwan University. His research interests are brain-computer interfaces and exoskeleton design.

measured EEG is in fact not an actual EEG, but a mixture of actual EEG and all other possible neurophysiological signals such as ocular artifact (i.e., EOG), muscle artifact (i.e., electromyography, EMG), and heart signals. While measuring the EEG signal, other neurophysiological signals are also measured simultaneously. The failure to produce a clean EEG signal significantly affects the $\mathrm{BCl}$ performance significantly. This paper focuses on techniques for artifact removal from the EEG and rendering the presented BCRS sufficiently for practical robust use.

Independent component analysis (ICA) has been proven to be an effective method for blind source separation (BSS), and has been widely used in the $\mathrm{BCl}$ community for separating artifacts from measured EEG signals. [2-5]. ICA treats the measured EEG signal as a linear combination of the actual EEG signal and other unknown source variables including artifacts and noises, and assumes that these sources are statistically independent. Based on this assumption, ICA decomposes $N$ measured EEG signals into $N$ independent signals (commonly known as the independent components, ICs) [6-8]. Among the ICs, some are actual EEG signals while the rest are artifacts or noises. The actual EEG signals are then reconstructed using the actual EEG components. While this method is easy to implement, it suffers from the problem that the artifact ICs must be manually selected and removed. Several studies have attempted to address this issue, with results including the regression method proposed by [9] which uses EOG as reference, while [10] and [11] respectively adopt scalp topography and sample entropy to remove artifact (EOG) ICs.

The BCRS is developed here to assist patients suffering from motor disabilities. To increase the accuracy of P300 detection and improve the automatic selection of clean EEG signals, an ICA-multiple kernel learning (ICA-MKL) algorithm is proposed. The proposed ICA-MKL is not only useful for P300 detection in our P300-based BCRS, but is also capable of detecting EOG artifacts in any motor-imagery-based $\mathrm{BCl}$. Therefore, the 4-class motor imagery data with eye-blink artifacts from the $2008 \mathrm{BCl}$ competition [12] is also used to test the effectiveness of ICA-MKL. In the motor imagery data, eye-blink artifacts are separated from measure EEG after ICA decomposition, and the artifact from eye-blink is automatically separated using the ICA-MKL. In the P300 data, the most important ICS are automatically extracted using ICA-MKL. The results shown in Section IV indicate that the proposed ICA-MKL method allows for automatic feature extraction and is able to effectively reject eye-blink artifacts from EEG signals. 


\section{P300 Data Collection and Experimental Setup}

\section{P300 Brain Controlled Rehabilitation System}

A Brain Controlled Rehabilitation System (BCRS) is developed based on the P300 potentials. Thus, the brain-robot interface consists of a P300-stimulus panel, an acquisition system for brain waves, and an intelligent P300 detection system. The BCRS used in our study is a $5 \times 5$ matrix consisting of 25 commands (see Figure 1) displayed in a liquid crystal display (LCD). The BCRS system is elicited by an oddball paradigm. Each row and column in the matrix is intensified (flashed) in a random order. Each intensification represents a visual stimulus and is presented for $100 \mathrm{~ms}$, with an inter-stimulus interval (ISI) of $75 \mathrm{~ms}$ between stimuli presentations. The onset of each intensification triggers an EEG time series of $500 \mathrm{~ms}$ called an epoch. In real practice, our system embeds a stimulus code into the EEG data stream in a time-locked fashion when a row or column intensification starts. Using the stimulus code, the recorded EEG data are split into epochs of $500 \mathrm{~ms}$. One round ends after each of the five rows and five columns is intensified once.

\begin{tabular}{ccccc}
\hline $\begin{array}{c}\text { Start } \\
\text { Position } \\
15\end{array}$ & Stop & $\begin{array}{c}\text { Isotonic } \\
\text { Exercise }\end{array}$ & $\begin{array}{c}\text { Isometric } \\
\text { Exercise }\end{array}$ & $\begin{array}{c}\text { Passive } \\
\text { Exercise }\end{array}$ \\
90 & 105 & 120 & 135 & 150 \\
$\begin{array}{c}\text { Flexion } \\
+0\end{array}$ & +20 & +40 & +60 & +80 \\
$\begin{array}{c}\text { Extension } \\
-0\end{array}$ & -20 & -40 & -60 & -80 \\
\hline
\end{tabular}

Figure 1. P300 panel and commands for rehabilitation exercises.

The user can control the rehabilitation robot through the panel. In the first row, "Start" and "Stop" are used to start the guidance of the rehabilitation robot and stop any exercise. There are three commands for exercise mode selection: "Isotonic Exercise", "Isometric Exercise", and "Passive Exercise". In the second and third rows, the commands represent the elbow angles. These commands are used to achieve position control for the isometric and passive ranges of exercise motion. In the fourth and fifth rows, the commands represent the percentages of maximum voluntary contraction. The force commands indicate the reference of the force control for the isotonic exercise. Through the commands in this panel, the user can control the BCRS to autonomously perform exercises.
During the round, the user focuses his or her attention on a specific character called a target character. Ideally, the P300 potential will be created in the EEG only if the row or column containing the target character is intensified. A simple decision rule can easily be formulated: the inferred character is the intersection of the row and column that elicits the P300 potential. However, in practice, the P300 potential may also be elicited in the EEG when rows or columns that do not contain the target character are intensified because characters other than the target one may also be within the user's field of view when he or she is looking at the target character. In this situation, the inferred character may not be the target one, and a robust P300 detector is required to prevent false-positives. Moreover, since the signal-to-noise ratio (SNR) for the EEG is very low, we improve the SNR by averaging epochs over a certain number of rounds.

\section{Experimental Setup and Preprocessing}

Since P300 potential is created in the central sites of the EEG measurements, the EEG is measured from the electrodes at the positions $\mathrm{Fz}, \mathrm{Fcz}, \mathrm{Cz}, \mathrm{CPz}, \mathrm{C} 3, \mathrm{C} 4, \mathrm{P} 7$, and $\mathrm{P} 8$ according to the $10-20$ system, where $\mathrm{P} 7$ and $\mathrm{P} 8$ are reference sites. The EEG potential measured from each channel is further subtracted from the average of the P7 and P8 potentials. Data are sampled at $250 \mathrm{~Hz}$. Each channel is band-pass filtered with upper and lower corner frequencies of 1 and $10 \mathrm{~Hz}$, respectively, using inverse discrete Fourier transform. The computer screen shows a $5 * 5$ matrix control panel, and the subjects were instructed to concentrate on the command words. The overall system scheme is shown in Figure 2[13], while Figure 3 shows the overall experimental setting.

To determine the performance of a brain-controlled rehabilitation system, three aspects must be considered.

\section{Data Collection}

The data collection procedure has three stages: subject preparation, training data collection, and test data collection.

\section{- Subject preparation}

Data from five voluntary, healthy subjects were recorded. All subjects sat on a fixed chair facing a 28-inch LCD at a distance of approximately 90 . To reduce impendence, an abrasive paste was applied to remove the upper dermal layer of the scalp. An electro-cap is then attached to the scalp, and an electrolyte gel is applied to the electrodes to further reduce impedance. Only impendences lower than $5 k \Omega$ are accepted. 


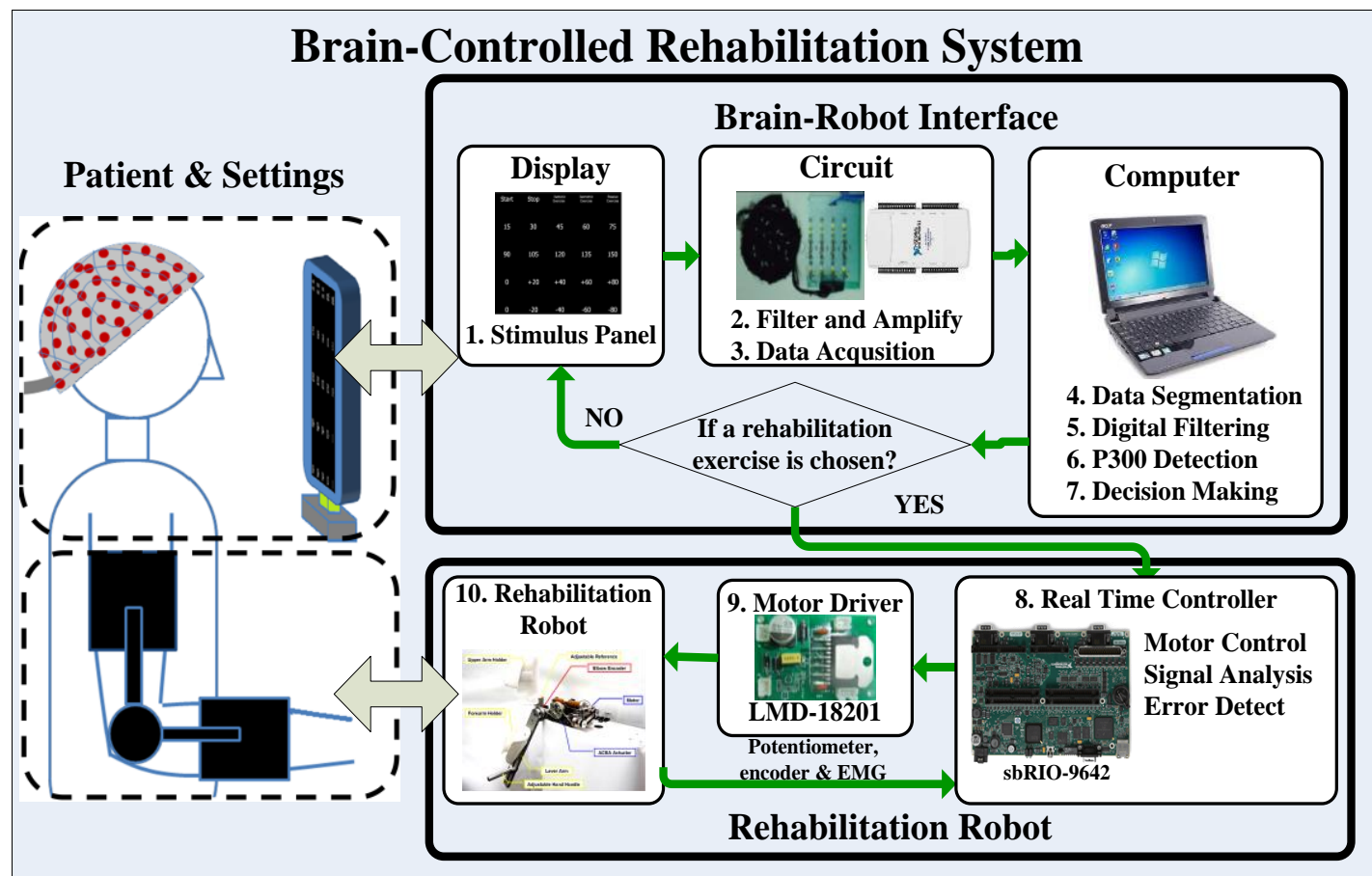

Figure 2. System architecture of P300 Brain-Controlled Rehabilitation System.

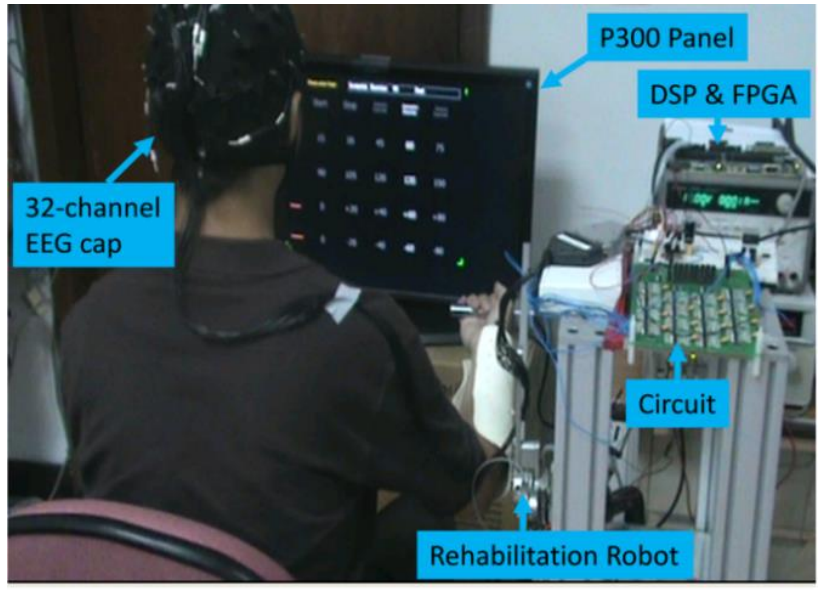

Figure 3. Experimental setting.

\section{- Training data collection}

The objective of this stage is to collect data for training a classifier that will be used during operation. $N_{t}$ different characters in the spelling matrix are randomly chosen as targets. A single trial consisting of $N_{r}$ rounds is performed for each target character. At the beginning of a trial, one of the chosen target characters is intensified for $4 \mathrm{~s}$, prompting the subject to focus his or her attention on that character. After the attention attracting intensification, a $2.5 \mathrm{~s}$ preparation gap is given, and all the characters in the matrix remain dark. Then, the $N_{r}$-round intensification process starts. The trial ends after the process is completed, and then the next trial starts. The training data collection stage stops when $N_{t}$ trials are finished.

In each round, there are 10 intensifications. If, for instance, the ith row is intensified in the $j$ th round $\left(j=1, \ldots, N_{r}\right)$, we can obtain the vector $x_{j}^{i}$ by concatenating the EEG epochs from different channels into the vector:

$$
x_{j}^{i}=\left[\left(x_{j 1}^{i}\right)^{T},\left(x_{j 2}^{i}\right)^{T}, \ldots,\left(x_{j k}^{i}\right)^{T}\right]^{T} \in R^{d}
$$

where $k$ is the number of channels ( $k=6$ in this study), and $x_{j k}^{i}$ is the EEG epoch recorded from the $k$ th channel. Since each epoch is $500 \mathrm{~ms}$ in length and the sampling rate is $250 \mathrm{~Hz}$, each epoch contains 125 samples. Therefore, the vector $x_{j}^{i}$ is of $d$ dimension, where $d=125 \times 6=750$. Since there are $N_{r}$ rounds per trial, the vector $x^{i}$, which is associated with the ith row intensification, is obtained by averaging the vectors $x_{j}^{i}$ over $N_{r}$ rounds, i.e., $x^{i}=\left(1 / N_{r}\right) \sum_{i=1}^{N_{r}} X_{j}^{i}$. Furthermore, one trial produces a total of 10 vectors. Five vectors are from rows and the other five vectors are from columns. If the vector $x$ is obtained from a row (or a column) that contains the target character after a single trial, the vector is defined as positive data with the label $y=1$; otherwise, it is negative data, and is labeled $y=-1$. According to this definition, the trial produces two positive data occurrences and 8 negative data occurrences. Finally, since $N_{t}$ trials are performed in a training session for a single subject, a training set composed of $N_{t} \times 10$ post-stimulus training data points of dimension $d$ is formed, in which $N_{t} \times 2$ are positive and the others are negative. The training set is used to train a P300 classifier, and the trained classifier is then used during operation to infer the target characters. 
Test data collection

Prior to the test stage, the experimenter asks the subject to remember a phrase composed of several words. During the testing stage, the subject tries to spell the phrase using the $\mathrm{P} 300 \mathrm{BCl}$. All the characters in the phrase are target characters. When the testing stage begins, the subject focuses on the first target character of the phrase. In the testing stage, each trial issues $n_{r}$ rounds, and the number of rounds $\left(n_{r}\right)$ in each trial in the testing stage is not necessarily the same as the $N_{r}$ in the training. Following the $n_{r}$ round intensification process - that is, after a single trial is complete -10 test data points are obtained, with each of the five rows and five columns in the spelling matrix generating a test data point. The classifier then starts to identify the most probable row and column that contain the P300 ERP. Once the indices of the most probable row and column are determined, the character that the subject was focusing on during the trial can be inferred for presentation on the LCD screen. The subject then focuses his or her attention on the next target character. Prior to the start of the next trial there is a rest period of $4 \mathrm{~s}$ during which no rows or columns are intensified. In addition, the testing stage differs from the training stage in that no attention-attracting intensification process is performed between consecutive trials.

\section{Data Extraction Using ICA-MKL}

\section{Independent Component Analysis}

ICA is a higher-order statistical method to identify the ICs with a non-Gaussian source signal. It assumes the observed signals (i.e., measured signals) $x(t)=\left\{x_{i}(t), i=1, \ldots, M\right\}$ are a linear combination of statistically independent sources (i.e., ICs) $s(t)=\left\{s_{i}(t), i=1, \ldots, M\right\}$. ICA supposes that the sources are unknown, and the number of sources is equal to the number of observers. The basic ICA model takes the form:

$$
x=A s,
$$

where $A$ is a $M \times M$ mixing matrix. ICA estimates the demixing matrix $W$ so that the ICs are obtained:

$$
s=W x
$$

The extended infomax ICA algorithm [14] by EEGLAB is used here to determine the demixing matrix.

\section{Multiple Kernel Learning}

In this study, three different kernels are chosen to implemet $M K L$, including the linear kernel

$$
K_{\text {linear }}\left(x_{i}, x_{j}\right)=x_{i}^{T} x_{j}
$$

the Gaussian kernel

$$
K_{\text {Gaussian }}\left(x_{i}, x_{j}\right)=\exp \left(-\frac{\left\|x_{i}-x_{j}\right\|_{2}^{2}}{2 \sigma^{2}}\right)
$$

and the exponential kernel:

$$
K_{E \times p}\left(x_{i}, x_{j}\right)=\exp \left(-\frac{\left\|x_{i}-x_{j}\right\|_{2}}{2 \sigma^{2}}\right) .
$$

The training set $\left\{\left(x_{i}, y_{i}\right)\right\}_{i=1}^{N}$ contains vectors $x_{i} \in X \subseteq \mathfrak{R}^{D}$ with labels $y_{i} \in\{-1,+1\}$. There are $M$ kernels in the MKL.

In the MKL, many kernels are combined and the kernel weight $\left(d_{m}\right)$ regulates the importance of the $\mathrm{mth}$ kernels. The kernels can be different. Here, one linear kernel, sixteen Gaussian kernels, and sixteen exponential kernels are chosen. The parameters of the Gaussian and Exponential kernels are chosen as $[0.01,0.05,0.1,0.5,1$, $2,5,7,10,12,15,17,20,25,40,100]$ and the parameters of the exponential kernels are chosen as $[0.01,0.05,0.1,0.5,1,2,5,7,10,12,15,17,20,25]$. The primary problem of MKL is formulated as below. Here, $K_{m}, m=1, \ldots, M$ are $M$ positive definite kernels. For any $m$, let $d_{m}$ be the kernel weight. $w_{m}$ is the norm vector of the separating hyperplane, $\alpha_{i}$ is the dual variable of SVM, $\xi_{i}$ is the slack variable for the $i$-th training sample, $b$ is bias term in SVM, and $C$ is a regular parameter between the margin cost and error cost.

$$
\begin{gathered}
\min _{\left\{f_{m}\right\}, b, \xi, d} \frac{1}{2} \frac{\left\|w_{m}\right\|_{2}^{2}}{d_{m}}+C \sum_{i}^{N} \xi_{i}, \\
\text { s.t. } y_{i} \sum_{m}\left\langle w_{m}, \Phi_{m}\left(x_{i}\right)\right\rangle+y_{i} b \geq 1-\xi_{i}, \forall i, \\
\xi_{i} \geq 0, \forall i \\
\sum_{m}^{M} d_{m}=1, d_{m} \geq 0, \forall m .
\end{gathered}
$$

The dual problem of the MKL is

$$
\max _{\alpha_{i}, \lambda} \sum_{i}^{N} \alpha_{i}-\lambda
$$




$$
\begin{gathered}
\text { s.t. } \sum_{i}^{N} \alpha_{i} y_{i}=0, \\
0 \leq \alpha_{i} \leq C, \forall i, \\
\frac{1}{2} \sum_{i, j}^{N} \alpha_{i} \alpha_{j} y_{i} y_{j} K_{m}\left(x_{i}, x_{j}\right) \leq \lambda, \forall m .
\end{gathered}
$$

The decision function of the MKL is given by

$$
f(x)=\sum_{i}^{N} \alpha_{i}^{*} \sum_{m=1}^{M} d_{m}^{*} K_{m}\left(x, x_{i}\right)+b^{*}
$$

Here, $\alpha_{i}^{*}, d_{m}^{*}, b^{*}$ are the optimal variables in the MKL and the SimpleMKL algorithm [9] is used to solve the MKL problem. SimpleMKL solves the MKL optimization problem using the reduced gradient method, which takes less computation time than other MKL methods.

\section{Automatic Feature Extraction by ICA-MKL}

The ICA-MKL algorithm for P300 detection is shown in Figure 4. The ICA-MKL is used to find the demixing matrix $\mathrm{W}$ and kernel weight vector $D$. Useful features are then extracted by multiplying $W$ and $D$. Finally, the useful features are used to train an MKL model as described above. The meaning of those symbols in P300 data are shown as below. $\quad X$ : $6^{*} 62500\{$ six channels*(five subjects*125 sample points* 100 data)\}; $\mathbf{x}$ : 6*12500\{six channels*(one subject*125 sample points* 100 data)\}; T: 500*125\{(five subjects*100 data)*125 sample points\}; t: $100 * 125$ \{(one subject*100 data)*125 sample points $\}$; demixing weights $\mathbf{W}=\left[\mathbf{w}_{1} ; \mathbf{w}_{2} ; \mathbf{w}_{3}\right.$; $\left.\mathbf{w}_{4} ; \mathbf{w}_{5} ; \mathbf{w}_{6}\right], \mathbf{w}: 1^{*} 6, \mathbf{W}: 6^{*} 6$; Kernel Weights $\mathbf{D}=\left[\mathrm{d}_{1} \mathrm{~d}_{2} \mathrm{~d}_{3} \mathrm{~d}_{4}\right.$ $d_{5} d_{6}$ ], D:1*6. P300 detection is a 2-class classification problem. In the motor imaginary data $(\mathrm{BCl} 2008$ data set $2 a)$, the problem is a four-class classification problem. The ICA-MKL algorithm can use the labels to automatically extract the useful data and reject artifacts for both applications.

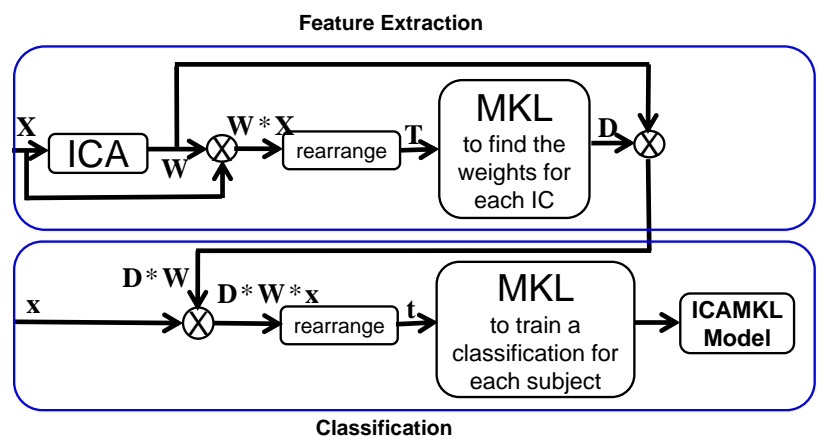

Figure 4. Feature extraction by ICA-MKL.

\section{Results and Discussion}

Figure 5 shows the P300 signals generated by averaging over 10 rounds and the wave forms of five subjects. Figure 6 shows the ICA signals generated by averaging 10 rounds and five subjects by ICA feature extraction. This process reveals that only one signal contains P300 signals.

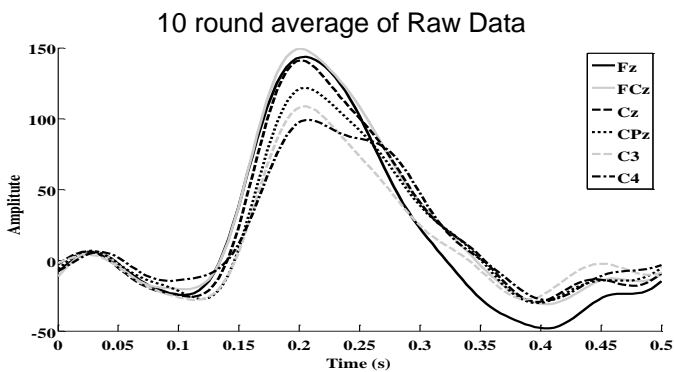

Figure 5. Average EEG.

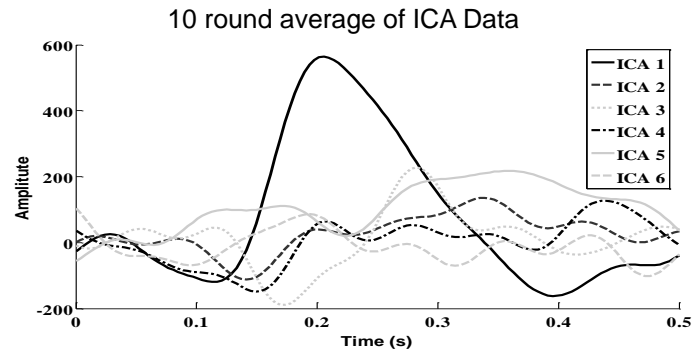

Figure 6. ICA results of 10-round average data.

\section{Results of ICA-MKL}

There are 100 training data points and 100 testing data points. Among the training/testing data, 50 contain P300 while the rest are non-P300 signals. Table I shows the inter-subject accuracy of MKL, and Table II shows the inter-subject accuracy of ICA-MKL. The average inter-subject accuracies are $84.4 \%$ and $86.2 \%$ for MKL and ICA-MKL, respectively. Using the ICA-MKL method, useful features can be easily found by an optimization procedure and artifacts can be automatically removed.

Table 1. Inter-subject results of MKL.

\begin{tabular}{|c|c|c|c|c|c|}
\hline Train & Subject1 & Subject2 & Subject3 & Subject4 & Subject5 \\
\hline Subject1 & 98 & 72 & 93 & 63 & 82 \\
\hline Subject2 & 95 & 97 & 96 & 88 & 83 \\
\hline Subject3 & 86 & 83 & 98 & 76 & 71 \\
\hline Subject4 & 87 & 92 & 96 & 94 & 78 \\
\hline Subject5 & 97 & 83 & 91 & 76 & 96 \\
\hline
\end{tabular}

Table 2. Inter-subject results of ICA-MKL.

\begin{tabular}{|c|c|c|c|c|c|}
\hline Train & Subject1 & Subject2 & Subject3 & Subject4 & Subject5 \\
\hline Subject1 & 98 & 79 & 95 & 71 & 91 \\
\hline Subject2 & 93 & 98 & 95 & 90 & 79 \\
\hline Subject3 & 95 & 86 & 100 & 78 & 80 \\
\hline Subject4 & 87 & 89 & 95 & 94 & 80 \\
\hline Subject5 & 97 & 81 & 92 & 73 & 97 \\
\hline
\end{tabular}




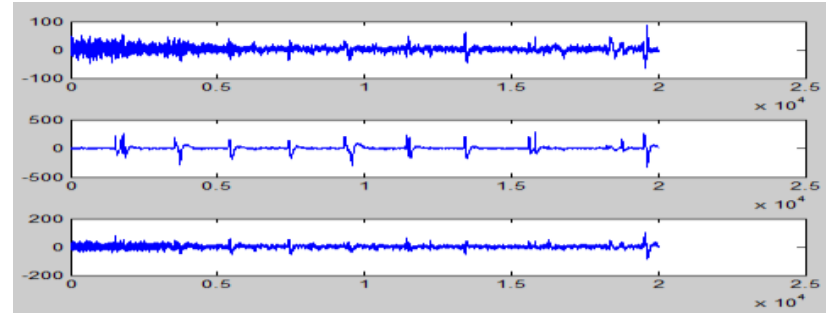

Figure 7. Three EOG artifact signals.

IC1

IC2

IC3

IC4

IC5

IC6

IC7

IC8

IC9

IC11

IC12

IC13

IC14

IC15

IC16

IC17

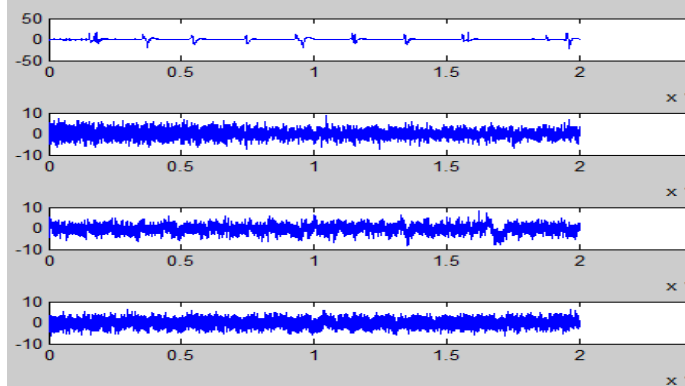

\section{$10^{4}$} $10^{4}$
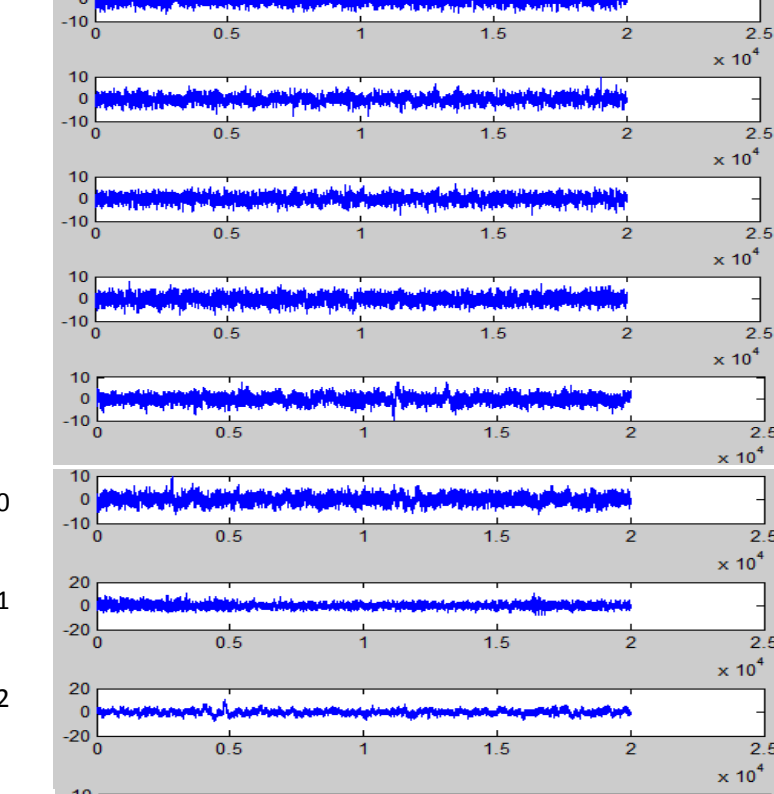

3

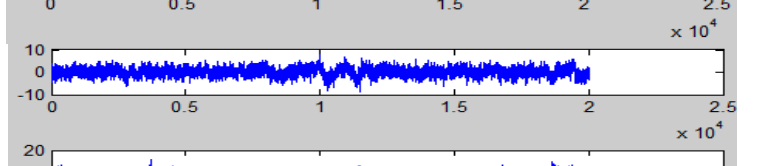

$-2$
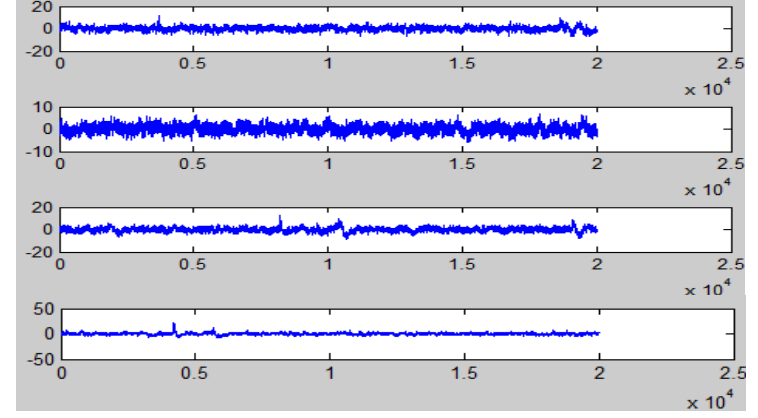

Figure 8. Rejected signals of $\mathrm{BCl} 2008$ Data 2a.

\section{Results on BCI 2008 Data 2 a}

$\mathrm{BCl} 2008$ Data 2a is used to test the performance of ICA-MKL. First, the ICs are extracted using ICA. We define a threshold to reduce the data dimension: if the
MKL weights for the ICs are lower than 0.05, the ICs are rejected. The remaining ICs are used to train the MKL model for classification. Shown below are the ICs from Subject 7 of the $\mathrm{BCl} 2008$ Data $2 \mathrm{a}$ to show the automatic artifact removing effect by ICA-MKL. Figure 7 shows three EOG signals and we can observe the EOG artifact inside the signal.

Figure 8 shows the ICs rejected by ICA-MKL. It is clear that IC1 represents an EOG artifact and the EOG artifact is automatically rejected by ICA-MKL. Figure 9 shows the ICs accepted by ICA-MKL. We can see that IC1 is not included in the ICs shown in Figure 8. We can also observe from Figure 8 that some EOG signals exist in IC20. The main difference between IC1 and IC20 is the mixing ratio of EOG to EEG: the mixing ratio for IC1 is obviously higher than that for IC20. Almost no EEG exists in IC1 while both EOG and EEG can be found in IC20. It can be concluded here that the proposed ICA-MKL method can reject ICs containing only artifacts. On the other hand, although IC20 also contains EOG, the EEG in IC20 also contains P300 potential. Therefore, accepting IC20 facilitates P300 detection.

IC22

IC23

IC24

IC25

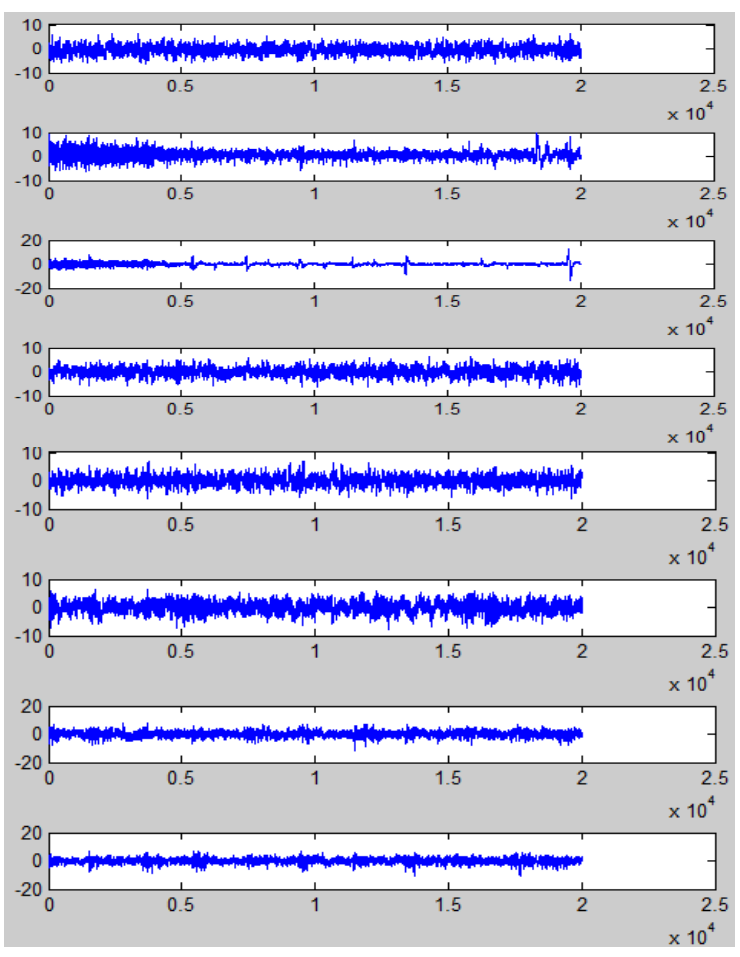

Figure 9. Accepted signals of $\mathrm{BCl} 2008$ Data 2a.

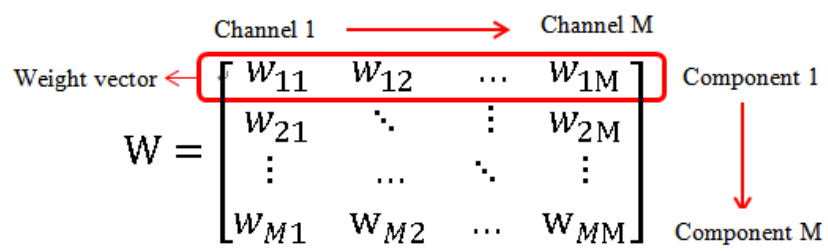

Figure 10. ICA demixing matrix. 


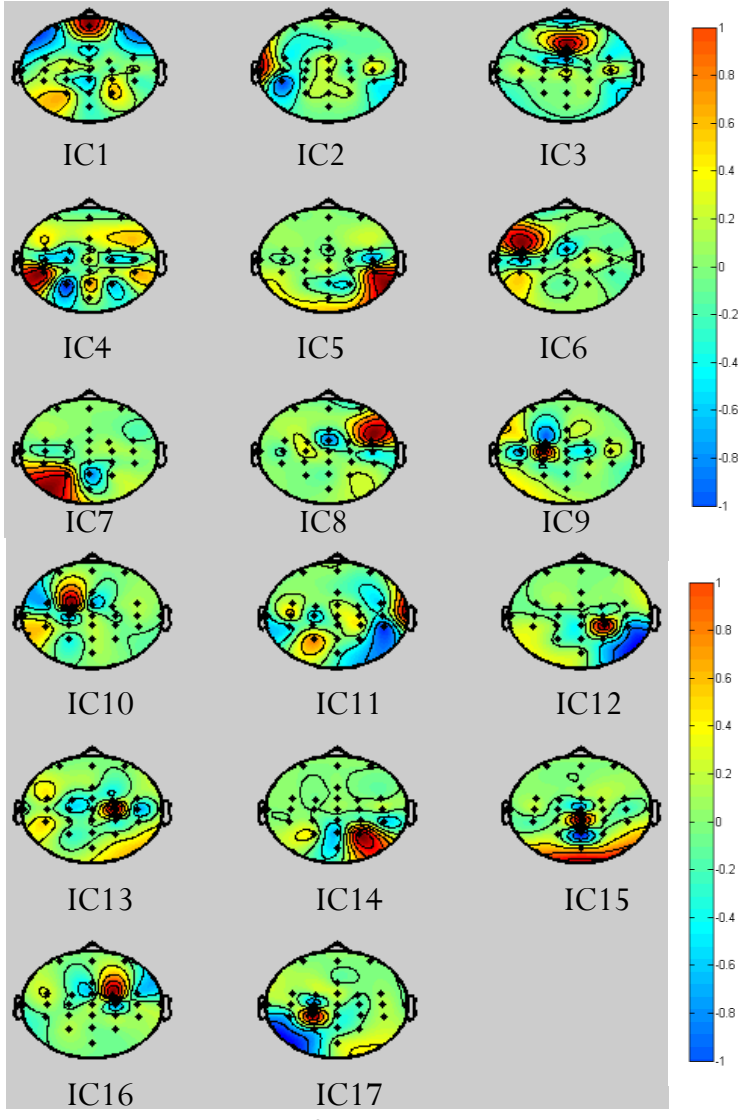

Figure 11. Rejected topology of $\mathrm{BCl} 2008$ Data 2a.

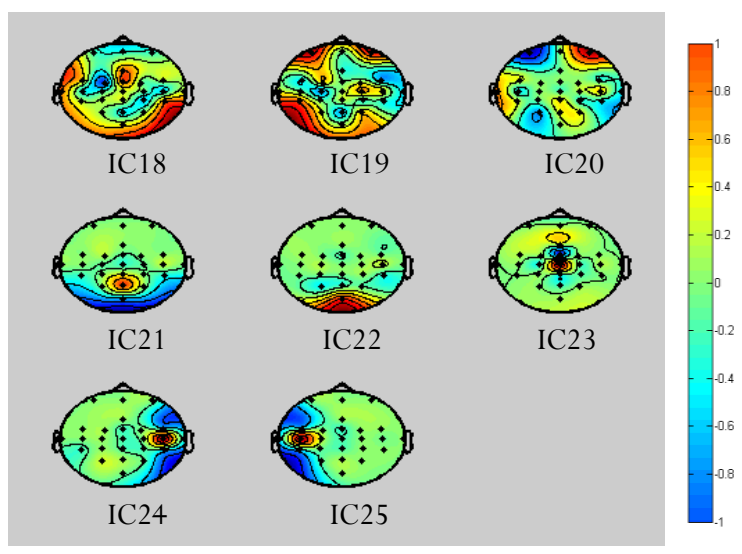

Figure12. Accepted topology of $\mathrm{BCl} 2008$ Data 2a.

After performing ICA on EEG signals, the measured EEG signals can be decomposed into ICs by using the obtained demixing matrix $W$. The components in the ith row of $W$ gives the weights of the scalp electrodes to the ith IC [10], as illustrated in Fig. 10. Thus, we can plot the scalp topography associated with each IC using the corresponding row components of the matrix $W$, as shown in Figure 11 and Figure 12. Moreover, it has been pointed out in [11] that the energy of eye-blink components should be distributed only over the front of the scalp while the energy of the EEG components is distributed over the whole scalp. Therefore, the vectors of scalp topographies are used as features for IC selection. However, according to our observations from Figure 11 and Figure 12, the energy region (i.e., the front of the scalp) of the eye-blink components cannot be observed from the topographies in practice. Therefore, scalp topography may not be a feasible approach to IC selection. On the contrary, our proposed ICA-MKL can automatically reject the eye-blink components without using scalp topography as a reference, thus being able to achieve more robust IC selection.

Finally, the comparison of kappa values on the $\mathrm{BCl}$ 2008 Data 2a set among different methods is provided in Table III. Overall, the proposed ICA-MKL method gives the best result on this data set, which should be attributed to the fact that this novel method can easily extract discriminating features. It is expected that better result can be obtained if more sophisticated signal processing and machine learning algorithms are employed after extracting the features via ICA-MKL.

Table 3. Comparison of the kappa value on $\mathrm{BCl} 2008$ Data 2a competition.

\begin{tabular}{|c|c|c|c|c|c|c|c|c|c|c|c|}
\hline$\#$. & Contributor & Kappa & 1 & 2 & 3 & 4 & 5 & 6 & 7 & 8 & 9 \\
\hline 1 & Kai Keng Ang & 0.57 & 0.68 & 0.42 & 0.75 & 0.48 & 0.40 & 0.27 & 0.77 & 0.75 & 0.61 \\
\hline 2 & Liu Guangquan & 0.52 & 0.69 & 0.34 & 0.71 & 0.44 & 0.16 & 0.21 & 0.66 & 0.73 & 0.69 \\
\hline 3 & Wei Song & 0.31 & 0.38 & 0.18 & 0.48 & 0.33 & 0.07 & 0.14 & 0.29 & 0.49 & 0.44 \\
\hline 4 & Damien Coyle & 0.30 & 0.46 & 0.25 & 0.65 & 0.31 & 0.12 & 0.07 & 0.00 & 0.46 & 0.42 \\
\hline 5 & Jin Wu & 0.29 & 0.41 & 0.17 & 0.39 & 0.25 & 0.06 & 0.16 & 0.34 & 0.45 & 0.37 \\
\hline & ICA-MKL & 0.529 & 0.65 & 0.44 & 0.57 & 0.54 & 0.42 & 0.28 & 0.73 & 0.62 & 0.51 \\
\hline
\end{tabular}

\section{Conclusion}

We have built a brain-controlled rehabilitation system (BCRS) and proposed an ICA-MKL method to further extract useful signals from the measured EEG. Results demonstrate that the method is not only able to extract useful P300 components but can also reject artifacts from the measured EEG, thus improving the usability of the P300-based BCRS. ICA-MKL also performs well on motor imaginary data, automatically removing eye-blink artifacts, thus achieving high classification accuracy for a 4-class motor imagery problem. In the future, we will evaluate the ICA-MKL methods through more practical clinical trials and conduct comparisons with other blind signal separation methods.

\section{Acknowledgements}

This work is partially supported by the National Science Council of the R.O.C. under grants NSC 100-2221-E-002-127-MY3 and NSC 100-2221-E-002-077MY3. 


\section{References}

[1] M. E. Neistadt, \& Crepeau, E. B. Willard and spackman's occupational therapy, 9th ed. Philadelphia: Lippincott Williams \& Wilkins, 1998.

[2] C. J. James and O. J. Gibson, "Temporally constrained ica: An application to artifact rejection in electromagnetic brain signal analysis," IEEE Transactions on Biomedical Engineering, vol. 50, no. 9, pp. 1108-1116, 2003. doi: $10.1109 /$ TBME.2003.816076

[3] W. Zhou, J. Zhou, H. Zhao, and L. Ju, "Removing eye movement and power line artifacts from the eeg based on ica," in 27th Annual International Conference of the Engineering in Medicine and Biology Society, 2005, pp. 6017-6020. doi: 10.1109/IEMBS.2005.1615863

[4] M. T. Akhtar, C. J. James, and W. Mitsuhashi, "Modifying the spatially-constrained ica for efficient removal of artifacts from eeg data," in 4th International Conference on Bioinformatics and Biomedical Engineering (iCBBE), 2010, pp. 1-4. doi: 10.1109/ICBBE.2010.5515306

[5] B. Azzerboni, M. Carpentieri, F. La Foresta, and F. C. Morabito, "Neural-ICA and wavelet transform for artifacts removal in surface EMG," in IEEE International Joint Conference on Neural Networks, 2004, vol. 4, pp. 3223-3228.

doi: $10.1109 /$ IJCNN.2004.1381194

[6] A. Kachenoura, L. Albera, L. Senhadji, and P. Comon, "Ica: A potential tool for bci systems," IEEE Signal Processing Magazine, vol. 25, no. 1, pp. 57-68, 2008.

doi: 10.1109/MSP.2008.4408442

[7] J. K. Hyvärinen, Erkki Oja, "Independent component analysis," John Wiley \& Sons,2001.

[8] L. Sun, Y. Liu, and P. J. Beadle, "Independent component analysis of EEG signals," in IEEE International Workshop on VLSI Design and Video Technology 2005, pp. 219-222. doi: $10.1109 /$ IWVDVT.2005.1504590
[9] C. W. Chen, C. C. K. Lin, and M. S. Ju, "Hand orthosis controlled using brain-computer interface," Journal of Medical and Biological Engineering, vol. 29, pp. 234-241, 2009.

[10] T. P. Jung, S. Makeig, M. Westerfield, J. Townsend, E. Courchesne, and T. J. Sejnowski, "Removal of eye activity artifacts from visual event-related potentials in normal and clinical subjects," Clinical Neurophysiology, vol. 111, no. 10, pp. 1745-1758, 2000. doi: $10.1016 / \mathrm{s} 1388-2457(00) 00386-2$

[11] C. P. Wang, H. P. Huang, Y. H. Liu, and T. H. Huang, "Automatic removal of eye-blink artifact in EEG based on ICA and SVDD," in Internatonal Conference on Service and Interactive Robotics, Taichung, Taiwan, 2011

[12] C. Brunner, R. Leeb, G. R. Müller-putz, and A. Schlögl, "BCl competition 2008 - graz data set a," ed, 2009.

[13] H. P. Huang, T. H. Huang, Y. H. Liu, Z. H. Kang, and J. T. Teng, "A brain-controlled rehabilitation system with multiple kernel learning," in IEEE International Conference on Systems, Man, and Cybernetics (SMC), 2011, pp. 591-596. doi: 10.1109/icsmc.2011.6083775

[14] T. W. Lee, M. Girolami, and T. J. Sejnowski, "Independent component analysis using an extended infomax algorithm for mixed subgaussian and supergaussian sources," Neural Computation, vol. 11, no. 2, pp. 417-441, 1999. doi: $10.1162 / 089976699300016719$ 